\title{
Advanced Intraundulator Electron Beam Diagnostics Using COTR Techniques*
}

\author{
A. H. Lumpkin, W. J. Berg, S. Biedron, M. Borland, Y. C. Chae, \\ R. Dejus, M. Erdmann, Z. Huang, K.-J. Kim, J. Lewellen, Y. Li, \\ S. V. Milton, E. Moog, D. W. Rule ${ }^{\dagger}$, V. Sajaev, and B. X. Yang
}

Advanced Photon Source, Argonne National Laboratory, Argonne, IL 60439

\begin{abstract}
A significant advance in intraundulator electron-beam diagnostics has recently been demonstrated based on coherent optical transition radiation (COTR) imaging. We find signal strengths from a microbunched beam in a UV-visible free-electron laser to be several orders of magnitude higher than that of incoherent optical transition radiation. In addition we report that the far-field images of COTR interferograms carry information about beam size and asymmetry, divergence, and pointing.
\end{abstract}

\section{INTRODUCTION}

One of the standard means of imaging electron beams is the use of optical transition radiation (OTR) as the conversion mechanism [1-4]. Its inherently good spatial resolution and ultrafast time response must be balanced with its lower conversion efficiency in some applications with bright beams. However, in the case of microbunched electron beams, we report a significant advance in intraundulator diagnostics based on the signal enhancements and structure observed in coherent optical transition radiation (COTR). Longitudinal microbunching of the electron beam occurs as it copropagates with the emitted synchrotron radiation within the undulator. The density modulation develops at the fundamental wavelength of the light, which leads to a growing fraction of electrons emitting in phase, or coherently. A favorable instability results in an exponential growth of the light generation in a self-amplified spontaneous emission (SASE) free-electron laser (FEL) [5-8]. The signal strength now goes as the square of the number of particles involved $\left(b_{n} N\right)^{2}$, where $b_{n}$ is the amplitude of the Fourier component of the electron distribution with spatial frequency $\mathrm{k}_{\mathrm{n}}$, and $\mathrm{N}$ is the total number of particles. We have routinely had to use neutral density (ND) filters providing attenuation factors of $10^{4}$ to $10^{5}$ (!) for COTR from 200-pC electron beams that have been microbunched. This would be an unheard of scenario with OTR imaging, and the light yield exceeds most scintillators.

In the case of the Advanced Photon Source (APS) FEL operating in the UV-visible regime, we have used standard CCD imaging cameras to obtain near-field, far-field, and spectral information on the COTR [9-12]. In addition to the unprecedented signal

* Work supported by U.S. Department of Energy, Office of Basic Energy Sciences under Contract No. W-31-109-ENG-38.
${ }^{\dagger}$ Carderock Division, NSWC, West Bethesda, MD 20817. 
strength in the near-field images (used to measure beam size), we have found noticeable structure and $\theta_{\mathrm{x}}-\theta_{\mathrm{y}}$ asymmetry in the far-field images plus narrow-band spectral emission. In our two-foil geometry, we see clear COTR interferometer images that are explained by the product of a bunch form factor (developed from the beam size) and the single electron interference pattern [13]. The spacings of the interference fringe peaks act like an internal "measurement grid" for beam size (as well as carrying information via fringe visibilities about beam divergence). For our optics we actually infer better beam size sensitivity $(\sigma \sim 30 \mu \mathrm{m})$ from the COTR fringe visibility than from the beam size image directly! A brief description of our experiments and some results to date will be presented.

\section{EXPERIMENTAL BACKGROUND}

These experiments were performed at the APS using beam accelerated to $217 \mathrm{MeV}$ by the S-band linac normally used as part of the injector system for the 7-GeV storage ring. We have obtained data with both the thermionic rf gun, with an 8-ns-long macropulse, and the photocathode (PC) rf gun, which generates a single micropulse at $6 \mathrm{~Hz}$. The guns $[14,15]$ and linac are described elsewhere. The beam is transported to the set of undulators (maximum of nine) in the low-energy undulator test line (LEUTL) tunnel [16]. A schematic of the experiment is given in Figure 1. It is not to scale, and there is an approximately 40-m transport line between the three-screen emittance station and the entrance of the undulators.

When all nine undulators are installed, the magnetic structure length is $21.6 \mathrm{~m}$. The properties of the undulators and diagnostic stations have been previously described [17]. Very briefly, the undulator cells have a period of $3.3 \mathrm{~cm}$ and length $\mathrm{L}=2.4 \mathrm{~m}$.

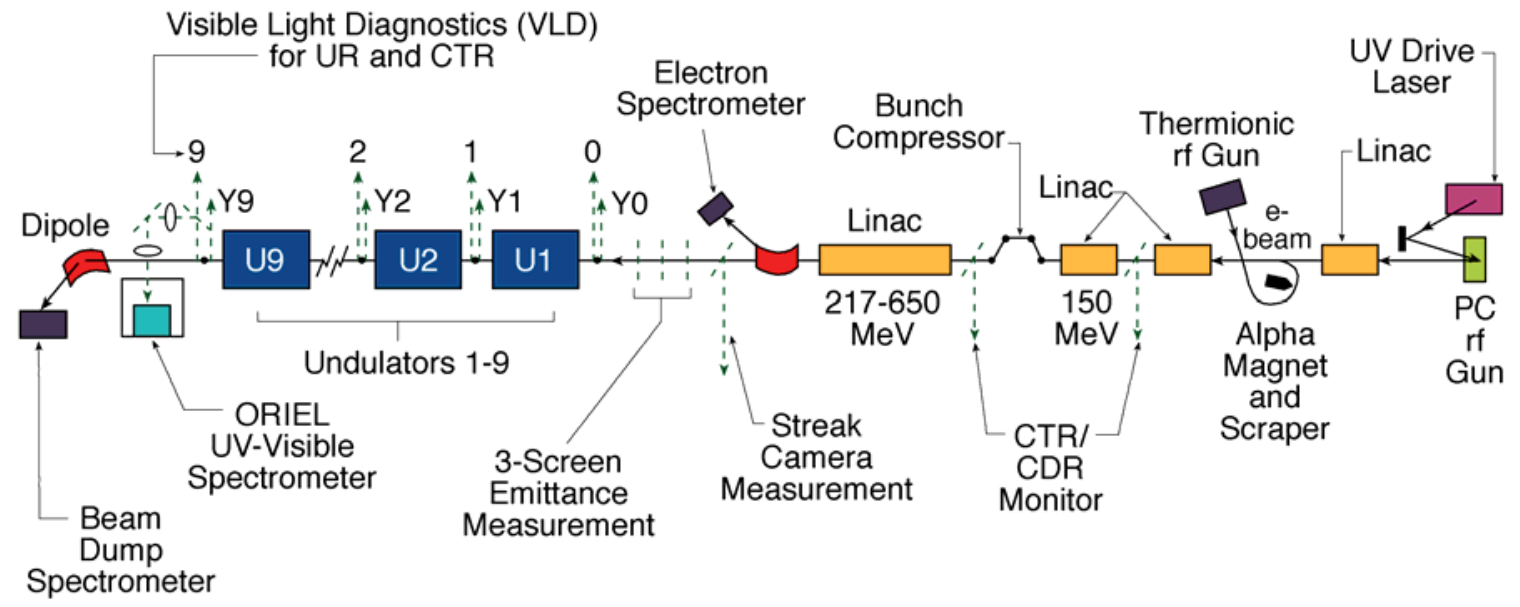

FIGURE 1. A schematic of the APS SASE FEL and the ten intraundulator diagnostic locations. 
They have a fixed gap with a field parameter $\mathrm{K}=3.1$. There is approximately a $0.38-\mathrm{m}$ space after each undulator. This space is used for diagnostics and focusing and steering elements before the first undulator and after each of the installed undulator sections. A schematic of these stations is shown in Figure 2. The screens on the first actuator include positions for a YAG:Ce/mirror, a mirror at $45^{\circ}$, and a thin $(6 \mu \mathrm{m}) \mathrm{Al}$ foil mounted with its surface normal to the beam direction. This thin foil serves two functions: (1) to block the stronger, visible undulator radiation (UR) and (2) to generate OTR or COTR as the e-beam transits the foil/vacuum interfaces. A digital camera views the e-beam images from the YAG and the reflected undulator radiation from the mirror. The second actuator, located $63 \mathrm{~mm}$ downstream, involves a retractable mirror at $45^{\circ}$ to the beam direction. Another digital camera and moveable lens provide both near-field and far-field (focus at infinity) imaging. This visible light detector (VLD) system thus provides both beam size and angular distribution data. Both neutral density (ND) filters and bandpass (BP) filters are selectable by up to three filter wheels in front of the cameras.

As indicated in Figure 2, a remotely controlled pick-off mirror and lens system can be used to redirect the UR or COTR to an Oriel UV-visible spectrometer. Spectral effects were observed including the onset of sideband production after FEL saturation. For the purposes here, it was important to verify the COTR narrow-band spectrum centered around the fundamental SASE wavelength. Chromatic effects in spatial focus or the time-domain are avoided with COTR.

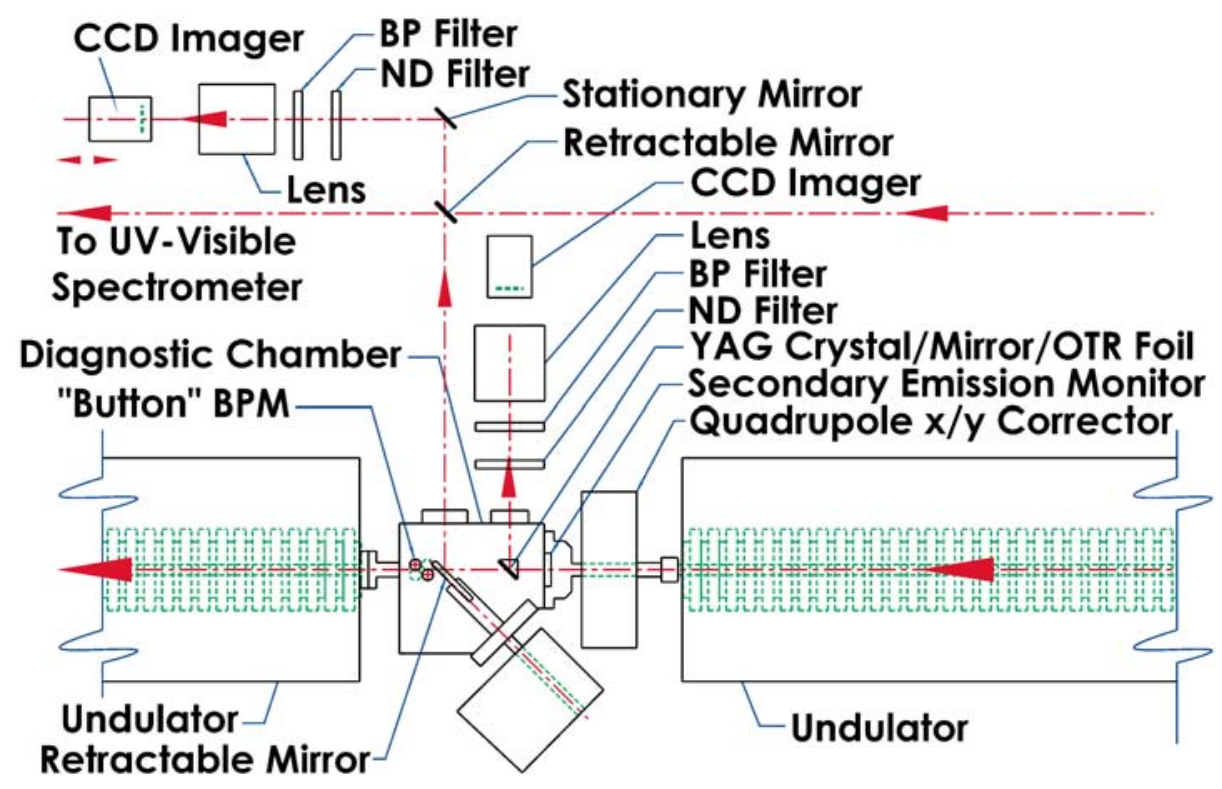

FIGURE 2. A detailed schematic of the intraundulator diagnostic stations showing the YAG actuator/cameras and the downstream $45^{\circ}$ pick-off mirror with the visible light detector (VLD) cameras. 


\section{EXPERIMENTAL RESULTS AND DISCUSSION}

We now present examples of results from the near-field imaging, far-field imaging, and the imaging spectrometer.

\section{Near-Field Imaging}

The near-field focus position of the lens provides beam size and profile information. In our geometry we can completely eliminate the competition from the dominant SASE light by using the blocking foil at the upstream position from the $45^{\circ}$ pick-off mirrors at each station. Examples of the $z$-dependent beam sizes are shown in Figure 3. In this case the FEL fundamental was at $530 \mathrm{~nm}$. We note that the needed ND value changed from 0 at VLD1 to 4.5 at VLD5, 6. A significant variation in the observed beam sizes is observed, in contrast to the expected constant beam sizes at the sampling points for a well-matched beam. Possible contributions to this effect are beam match into the undulators, beam image size reductions due to COTR, a transverse dependence of the bunching fraction coupled with the COTR effect, chromatic effects on lens focus for broadband OTR versus narrow-band COTR, and a camera focus error in the early stations. It is noted that we believe the major contributions are the first three. Since we see small beam structures in the VLD0 and VLD1 cameras on some shots out of the 100 images, we believe focus effects are minimal.

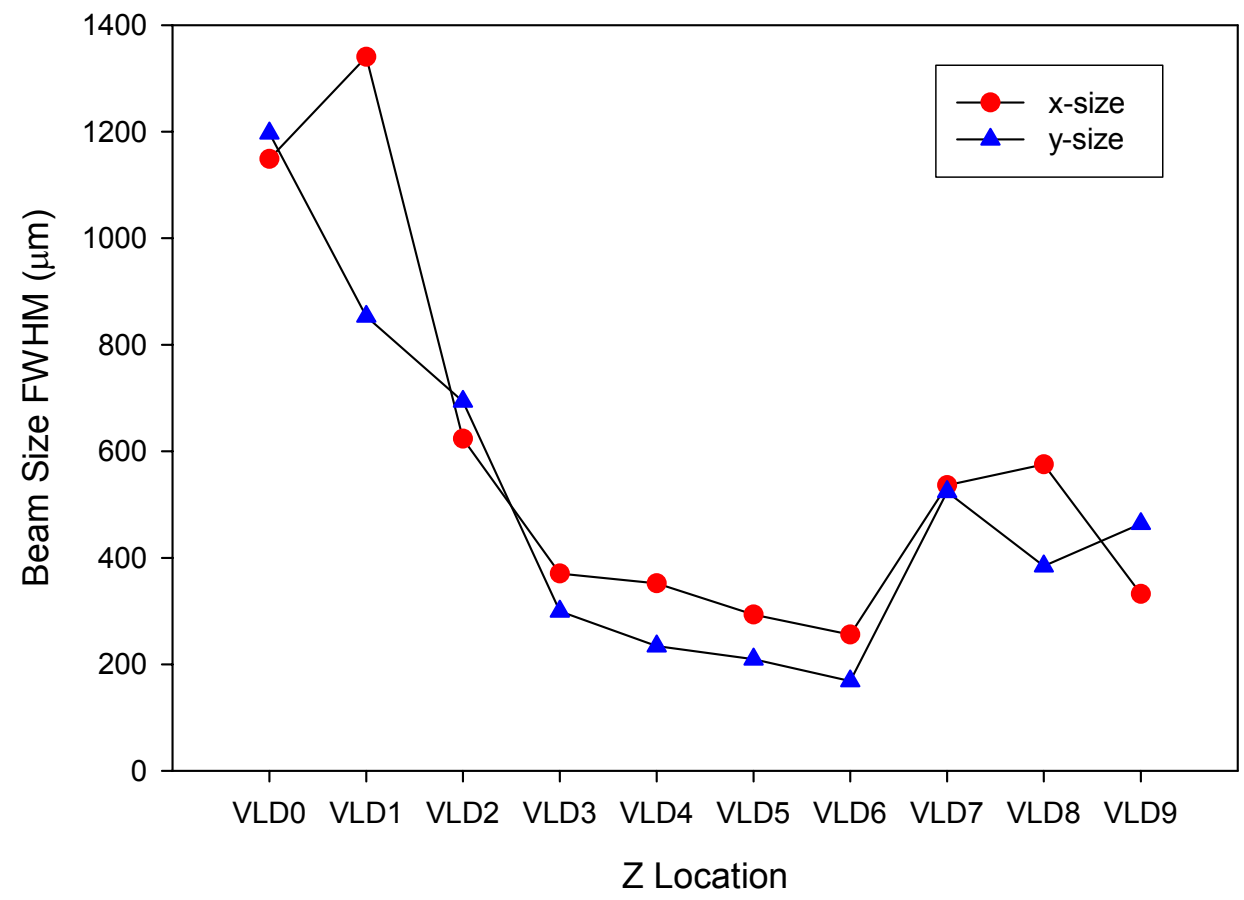

FIGURE 3. An example of $z$-dependent, $\mathrm{x}$ - and $\mathrm{y}$-beam sizes observed in the undulator areas. These data are from March 30, 2001. 
We have performed a calculation of the beam spot narrowing that occurs when COTR is used as the conversion mechanism. If the bunching fraction is assumed to be uniform across the Gaussian transverse dimension then the $\left(b_{n} N\right)^{2}$ term for the peak intensity will approximately give a $\sqrt{ } 2$ narrowing of the "observed" peak. We have experimentally tested this simple model by taking data without (COTR) and with (OTR) a 500-nm short pass filter, which would attenuate by 100 all wavelengths greater than about $520 \mathrm{~nm}$. An example of this effect is shown in Figure 4. The horizontal beam size observed when significant gain has occurred starting at VLD5 is narrower when the bandpass filter is not used (COTR). The incoherent OTR source provides the actual e-beam size. We note that at VLD8 the single filter is insufficient to block the entire strong microbunching signal, so clean OTR imaging is not achieved for this point.

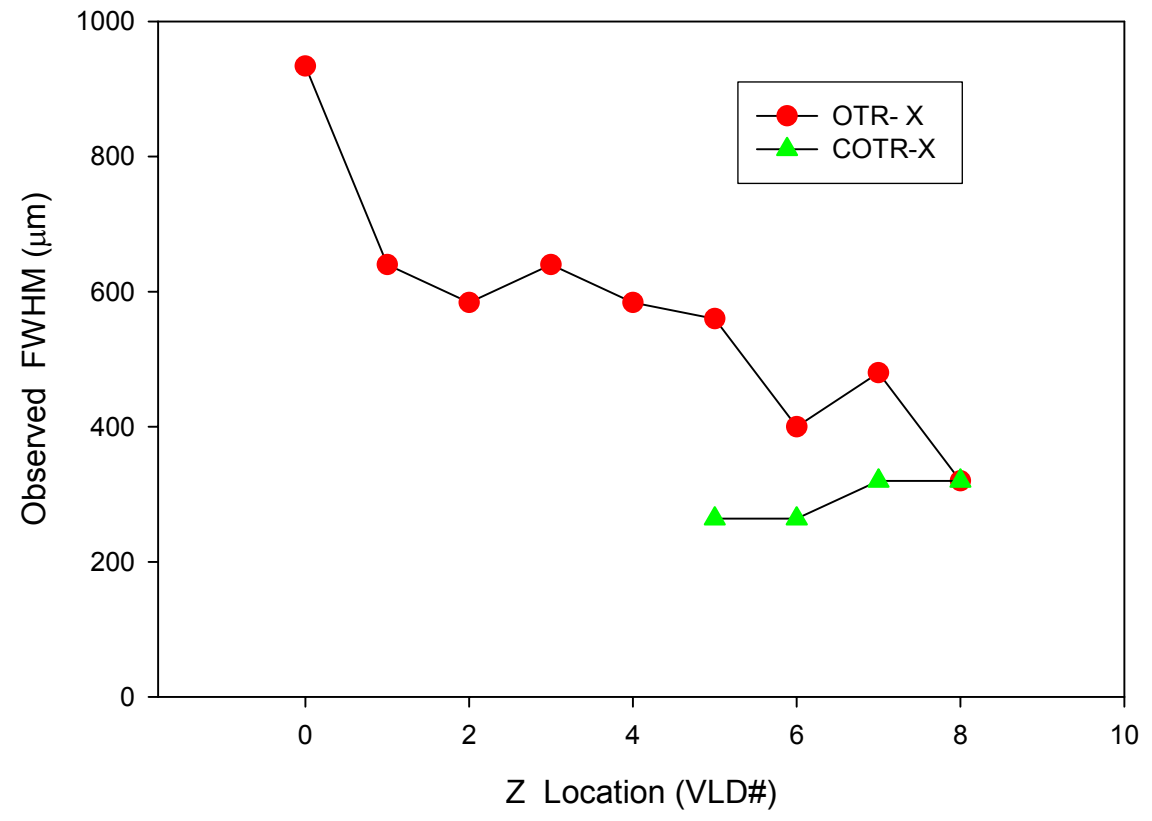

FIGURE 4. An example of $z$-dependent $x$-beam sizes observed with (OTR) and without (COTR) the 500-nm short pass filter at VLD5 - VLD8. Data are from December 20, 2001.

\section{Far-Field Imaging}

The camera-to-lens $z$ separation can be remotely controlled by the stepper motor. For far-field imaging angular distribution patterns are obtained. Based on the analytical model described in Ref. 13, the single-electron OTR interference (OTRI) pattern for two sources $63 \mathrm{~mm}$ apart is first calculated. This includes effects due to beam divergence. For example, we estimate the beam scattering from the first foil as about 0.1 to $0.2 \mathrm{mrad}$ at $220 \mathrm{MeV}$. The calculated OTRI pattern exhibits fringes at $\pm 1.9, \pm 4.6, \pm 6.3$, and $\pm 7.5 \mathrm{mrad}$. The bunch form factor is multiplied times this pattern. Large beam $(>100 \mu \mathrm{m})$ sizes have very narrow form factor functions in $\theta$ space. Figure 5 shows an example of the COTR fringe visibility variation with beam size. The COTR second fringe peaks are visible for beam sizes less than $50 \mu \mathrm{m}$. The observed first fringe peak position varies rapidly for $\sigma>100 \mu \mathrm{m}$. An example of an 
image is given in Figure 6. In this case the initial beam size asymmetry in $\sigma_{x}$ and $\sigma_{y}$ results in the single peaks in $\theta_{\mathrm{x}}$ and multiple peaks in $\theta_{\mathrm{y}}$. By looking at the fringe peak relative intensities for $\sigma_{\mathrm{y}}=15,20$, and $30 \mu \mathrm{m}$, the $\theta_{\mathrm{y}}$ pattern was found to be consistent with a beam size of $30 \mu \mathrm{m}$ and total divergences of about $0.2 \mathrm{mrad}$. The $\sigma_{\mathrm{y}}$ $=30 \mu \mathrm{m}$ result is smaller than the limiting resolution in the optical system of about one pixel at $80 \mu \mathrm{m} /$ pixel for near-field focus. Further studies are needed in this area to develop accuracy on the beam size. In addition, we have reported elsewhere the sensitivity of the symmetry of the $\pm \theta_{\mathrm{y}}$ peaks to e-beam steering with the correctors [18].

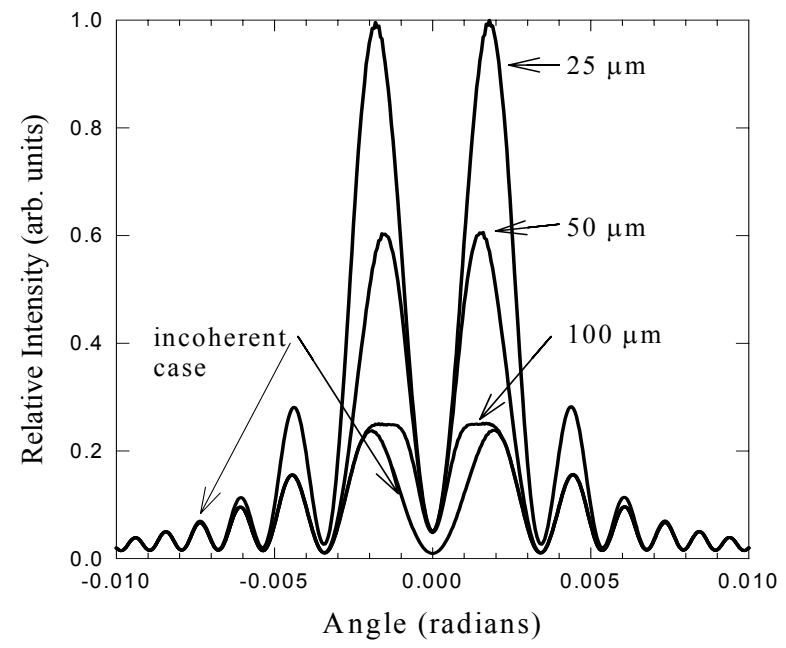

FIGURE 5. A comparison of COTR interference fringe visibility for different beam sizes, $\sigma_{\mathrm{y}}=25,50$, and $100 \mu \mathrm{m}$.

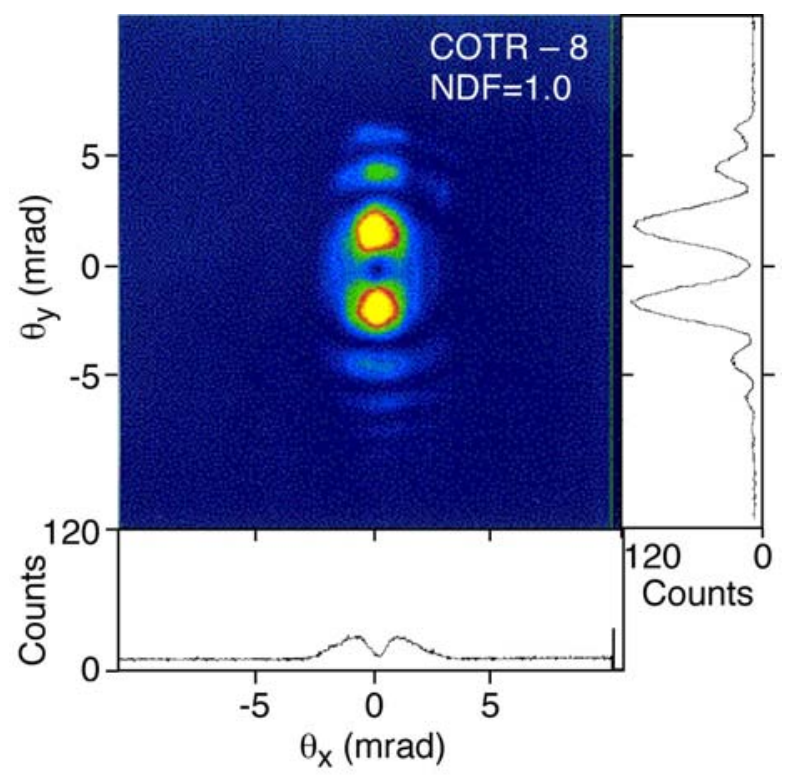

FIGURE 6. An example of a COTR interference image taken after undulator 8 in a case when this was post-FEL saturation. The clear $\theta_{\mathrm{x}}-\theta_{\mathrm{y}}$ asymmetry and the fringe visibility support corresponding beam sizes of $\sigma_{\mathrm{x}} \approx 100 \mu \mathrm{m}$, and $\sigma_{\mathrm{y}} \approx 30 \mu \mathrm{m}$, respectively. 


\section{Imaging Spectrometer}

The expected microbunching at the fundamental wavelength is clearly illustrated in a spectrum from one of our early studies [19]. As shown in Figure 7, the SASE FEL peak and the COTR peak overlap in wavelength at $530 \mathrm{~nm}$. The COTR is a little broader in this example taken after undulator 5 . The electron beam energy jitter can be observed directly in the wavelength jitter of the images. As a reference the GreNe calibration line is shown at $543.5 \mathrm{~nm}$, and its width indicates operational resolution of about $0.8 \mathrm{~nm}(\sigma)$ for these conditions.

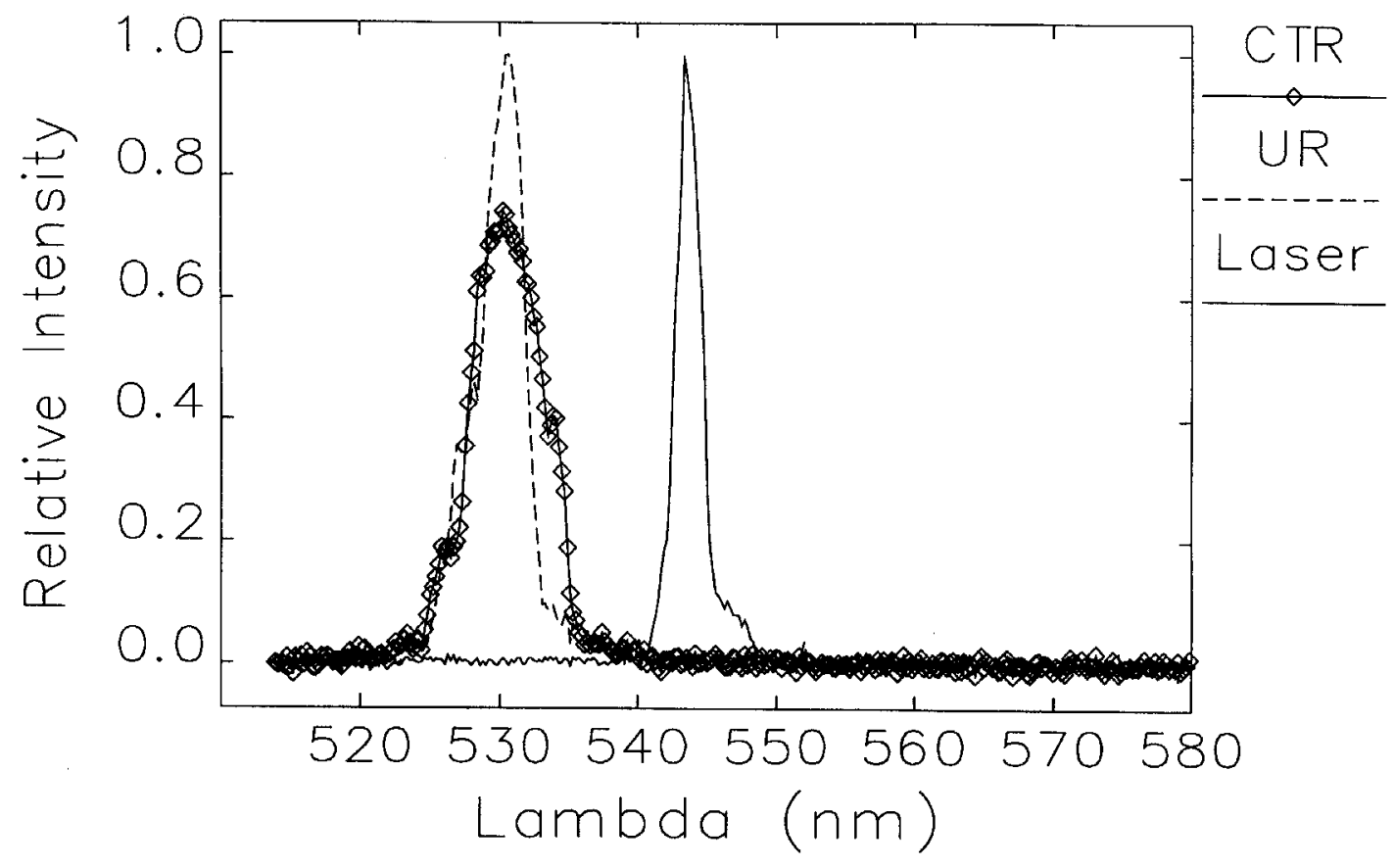

FIGURE 7. A comparison of the SASE FEL spectrum, the COTR spectrum, and the calibration laser signal obtained after undulator 5. The discrete COTR line is quite different from the broadband OTR spectrum.

\section{SUMMARY}

In summary, the COTR imaging techniques provide all the advantages of OTR imaging with the additional features of signal enhancements up to $10^{5}$ and monochromatic spectral output. These features provide a near-ideal, intraundulator electron-beam characterization capability. We hope to extend the techniques to the VUV in the coming year as the APS FEL project pushes to operate at $130 \mathrm{~nm}$. 


\section{ACKNOWLEDGEMENTS}

The authors acknowledge the support of Om Singh, Antanas Rauchas, Efim Gluskin, and Rodney Gerig of the APS.

\section{REFERENCES}

1. Wartski, L. et. al., J. Appl. Phys. 46, 3644 (1975).

2. Rule, D. W., Nucl. Instrum. Methods in Phys. Res. B24/25 901 (1987).

3. Lumpkin, A. H. et al., NIM A296, 150 (1990), D. W. Rule et al., Idem, p. 739 (1990).

4. Lumpkin, A. H. et al., Nucl. Instrum. Methods in Phys. Res. A429, 336 (1999).

5. Derbenev, Y. S., Kondratenko, A. M., and Saldin, E. L., Nucl. Instrum. Methods A193, 452 (1982).

6. Orzechowski, T. J. et al., Nucl. Instrum. Methods A250 144 (1986).

7. Hogan, M. et al., Phys. Rev. Lett. 81, 4897 (1998).

8. Milton, S. V. et al., Phys. Rev. Lett., 85988 (2000).

9. Tremaine, A. et al., Phys. Rev. Lett. 81, 5816 (1998).

10. Lumpkin, A. H. et al., Phys. Rev. Lett. 86, 79 (2001).

11. Lumpkin, A. H. et al., "Evidence for Microbunching "Sidebands" in a Saturated FEL Using Coherent Optical Transition Radiation," accepted in Phys. Rev. Lett. March 2002.

12. Lumpkin, A. H. et al., "Comprehensive z-Dependent Measurements of Electron-beam Microbunching using COTR in a Saturated SASE FEL," Nucl. Instrum. Methods in Phys. Res. (in press).

13. Rule, D. W., and Lumpkin A. H., Proc. of the IEEE 2001 Particle Accelerator Conference, Chicago, IL, pp. 1288-1290 (2001).

14. Lewellen, J. W. et al., Proc. of the 1998 Linac Conference, Chicago, ANL-98/28, Vol. 2, pp. 863-865 (1999).

15. Biedron, S. et al., Proc. of the IEEE 1999 Particle Accelerator Conference, New York, NY, pp. 2024-2026 (1999).

16. Milton, S. V. et al., Nucl. Instrum. Methods in Phys. Res., A407, 210 (1998).

17. Gluskin, E. et al., Nucl. Instrum. Methods in Phys. Res., A429, 358 (1999).

18. Lumpkin, A. H. et al., Proc. of the 2001 Particle Accelerator Conference, Chicago, IL, pp. 550-552 (2001).

19. Lumpkin, A. H. et al., "Utilization of CTR to Measure the Evolution of Electron-Beam Microbunching in a SASE FEL," Nucl. Instrum. Methods in Phys. Res. (in press). 